\title{
Clinicopathological and prognostic significance of Twist overexpression in NSCLC
}

\author{
Meng Li ${ }^{1,2, *}$, Xing Zhang ${ }^{1,2, *}$, Xiaoqing $\mathrm{Xu}^{1,2}$, Jiubin $\mathrm{Wu}^{3}$, Kaiwen $\mathrm{Hu}^{4}$, Xiuwei Guo ${ }^{1}$ \\ and Peitong Zhang ${ }^{1}$ \\ ${ }^{1}$ Department of Oncology, Guang'anmen Hospital, China Academy of Chinese Medical Sciences, Beijing, China \\ ${ }^{2}$ Beijing University of Chinese Medicine, Beijing, China \\ ${ }^{3}$ Department of Traumatology and Orthopedics, First Affiliated Hospital of Tianjin University of TCM, Tianjin, China \\ ${ }^{4}$ Department of Oncology, Dongfang Hospital, Beijing University of Chinese Medicine, Beijing, China \\ *These authors contributed equally to this work
}

Correspondence to: Peitong Zhang, email: peitong_zhang@163.com

Keywords: non-small cell lung cancer; Twist; prognosis; meta-analysis

Received: August 29, 2017 Accepted: November 14, 2017 Epub: February 14, 2018 Published: March 06,2018

Copyright: Li et al. This is an open-access article distributed under the terms of the Creative Commons Attribution License 3.0 (CC BY 3.0 ), which permits unrestricted use, distribution, and reproduction in any medium, provided the original author and source are credited.

\section{ABSTRACT}

Several studies were conducted to explore the prognostic significance of Twist in non-small cell lung cancer (NSCLC), however, contradictory results in different studies were reported. To this end, we presented a systematic review aiming to summarize the prognostic significance of Twist in patients with NSCLC. 5 studies involving a total of $\mathbf{5 7 2}$ patients were identified. The result indicated that high Twist expression was significantly associated with a worse overall survival $(O S)$ (hazard ratio $(H R)=2.19$, $95 \%$ confidence interval $(95 \% \mathrm{CI})=1.64-2.94, p<0.001 ; \mathrm{I}^{2}=0.0 \%$, fixed effect $)$, recurrence-free survival (RFS) $(H R=2.476,95 \%$ CI $=1.728-3.547, p<0.001$; $I^{2}=0.0 \%$, fixed effect) and lymph node or other metastasis (odds rate $(O R)=0.419$, $95 \% \mathrm{CI}=0.259-0.679, P<0.001$, fixed effect). Subgroup analysis revealed that the expression of Twist in Chinese patients might be more closely associated with the prognosis of NSCLC than in American patients. Overall, these results indicated that Twist over-expression in patients with NSCLC might be related to poor prognosis and serves as an unfavorable predictor of poor clinicopathological prognosis factor.

\section{INTRODUCTION}

Primary lung cancer is the leading cause of cancer mortality worldwide among both men and women $[1,2]$, and non-small cell lung cancer (NSCLC) accounts for about $80 \%$ of all lung cancer [3]. The high recurrence and metastasis largely contribute to the poor overall prognosis [4]. Thus, the lack of major improvement in the 5-year survival rate of NSCLC has driven the search for new strategies aiming at identifying specific biological markers of metastases to predict the prognosis in patients.

Local invasion can be considered as an initial step in the malignancy of carcinomas, leading to the generation of distant metastasis. Epithelial to mesenchymal transition (EMT) is an epithelial cell into a mesenchymal cell with the ability to invade and migrate adjacent tissues. It is a recent discovered cellular process in a wide variety of cancer cells, and it enhances the potential of tumor invasion, metastasis and chemoresistance $[5,6]$. One of the hallmarks of EMT is the functional loss of E-cadherin, which is currently thought to be a suppressor of invasion during carcinoma progression [7]. Indeed, transcriptional repression has emerged as a fundamental mechanism for repressing E-cadherin during tumour progression. As one of multifunctional transcription factors that dynamic silences E-cadherin, Twist is now thought to be involved in tumour progression, thus having potential clinical interest $[8,9]$. The two Twist-like proteins, Twist- 1 and Twist-2 (formerly Dermo-1), belong to the basic helix-loop-helix (bHLH) family and have been identified to promote EMT [10-13]. Well conserved during evolution, two Twist genes exist in vertebrates and share high structural homology. [14] These two proteins display a high degree of sequence similarity in their $\mathrm{C}$-terminal half and are 
more divergent in their $\mathrm{N}$ terminus. When Twist-2 lacks a glycine-rich region that presents in Twist-1 [15]. While both are silent in most healthy adult tissues, they were found overexpressed in various types of human tumors including a variety of carcinomas as well as sarcomas, melanomas, gliomas and neuroblastomas [16]. Twist-1 and Twist-2 do not display major transforming capabilities but still cooperate with activated oncoproteins in promoting EMT. [14] In the course of tumor progression, reactivation of EMT inducing transcription factors, Twist-1 and Twist-2, trigger the generation of cells with self-renewal capabilities, favoring both the growth of the primary tumor and the initiation of secondary tumors following cell dissemination. While, in most articles, Twist is also known as Twist-1 [9]. Although Twist-1/2 was included when literature searching in this article, actually, Twist-1 was discussed by carefully reading the finally involved five articles. Twist-1(Twist) overexpression has been reported in a variety of epithelial cancer cells with clinical correlations with poor prognosis, an event associated with its capacity to promote EMT [16-19] and these malignancies include breast cancer [20], colorectal cancer [21], pancreatic ductal adenocarcinoma [22] and oral cancer [23]. However, the prognostic value of Twist in lung cancer remains controversial. Some studies [2426] have suggested that the expression of Twist might not predict the survival of NSCLC, while the other researches [27-30] have indicated a specific correlation between Twist expression and patient prognosis. Besides, the research of Hung et al. [31] suggested Twist was associated with a shorter overall survival (OS), whereas the overexpression of Twist did not influence recurrencefree survival (RFS). We therefore carried out a systematic review of published literatures to quantitatively analyze the prognostic value of Twist in NSCLC patients based on current evidence.

\section{RESULTS}

\section{Literature search}

The literature search strategy and study selection are summarized in Figure 1. A total of 85 papers were identified of which 31 duplicative papers were excluded. As for the remaining 54 papers, 40 were excluded by scanning either the title or abstract. For the 14 remaining potentially related studies, the full-text was carefully read. 8 were excluded for insufficient data to assess the hazard ratios (HRs) of prognosis outcomes, and 1 was excluded for not focusing on this topic. Although evaluating the estimated HRs from the survival curves might be a reliable and the only feasible approach $[32,33]$, however, we found major deviations in two papers $[34,35]$ extracted from the same study of Pallier K et al. [36]. Thus, this study [36] failed to directly reveal the HRs and 95\% confidence interval $(95 \% \mathrm{CI})$ from the original data was excluded. At last, 5 studies involving 572 patients were eligible for this meta-analysis [27-31].

\section{Characteristic of included studies}

As listed in Table 1, the five included studies contained 572 patients. As for assessment of included studies, the Newcastle-Ottawa Quality Assessment Scale (NOS) of four included studies was 7 and one included study was 6 (Supplementary Table 1). Out of the five studies, four evaluated patient cohorts proceeded in China, and one was from America. Three studies applied OS and RFS as outcomes to explore prognosis correlation with Twist expression; the other two studies considered OS as the only outcome. Three studies demonstrated that Twist overexpression was an independent prognostic factor $(P$ $<0.05)$ [27, 28, 30], while Hui et al. [29] suggested an inverse correlation between Twist expression and patient prognosis by a multivariate Cox regression analysis $(P>$ 0.05). Besides, another study [31] suggested Twist was associated with a shorter OS instead of RFS. Four studies reported the follow-up time (range, 3 to 95 months), while the other one did not report the follow-up time [27]. In addition, the sample size was different, varying from 75 patients to 153 patients.

\section{Association of twist with survival}

\section{Meta-analysis of OS}

Five studies involving 572 patients were included in the meta-analysis of OS. As shown in Figure 2, in view of the significant heterogeneity $\left(\mathrm{I}^{2}=0.0 \%, p=0.488\right)$, the fixed-effect model was used. A significant correlation between the expression of Twist and OS was observed $(\mathrm{HR}=2.19,95 \% \mathrm{CI}=1.64-2.94, p<0.001)$, and the result revealed that overexpression of Twist predicted worse OS when compared with the low expression of Twist.

\section{Subgroup meta-analyses}

Table 2 shows the subgroup meta-analyses. All pooled HRs were obtained by using a fixed-effect model. Three studies reporting the RFS of patients with NSCLC were all included into the meta-analysis. As shown in Figure 3 and Table 2, a distinct correlation was observed between the Twist and RFS ( $\mathrm{HR}=2.476,95 \% \mathrm{CI}=$ $1.728-3.547, p<0.001)$, with heterogeneity $\mathrm{I}^{2}=0.0 \%(p$ $=0.414$ ). Poor prognosis was found in NSCLC with Twist overexpression under univariate analyses (pooled $\mathrm{HR}=$ $3.219,95 \% \mathrm{CI}=1.826-5.674, p<0.001)$ and multivariate analyses (pooled HR $=1.877,95 \% \mathrm{CI}=1.268-2.779$, $p=0.002$ ). Results showed that in terms of country, unfavorable prognosis was found in China (pooled HR = $2.235,95 \% \mathrm{CI}=1.619-3.086, p<0.001)$. Among the study with follow-up, unfavorable survival results were obtained whether the follow-up time was longer than 36 months or 
Table 1: Characteristics of the included studies

\begin{tabular}{|c|c|c|c|c|c|c|c|c|c|}
\hline study & Country & $\begin{array}{l}\text { Sample } \\
\text { size }(n)\end{array}$ & $\begin{array}{c}\text { Histology } \\
\text { type }(\mathrm{AC} \%)\end{array}$ & $\begin{array}{c}\text { Twist } \\
\text { positive (\%) }\end{array}$ & Outcome & Cut-off & Analysis & Follow-up (M) & $\begin{array}{l}\text { recruitment } \\
\text { time }\end{array}$ \\
\hline Hung, J. J, 2009 & China & 87 & $62.10 \%$ & 36.8 & RFS/ OS & Positive $>50 \%$ & $\mathrm{U}$ & Median43.2 (20 to 52.2) & 2003-2004 \\
\hline Jiang W, 2012 & China & 137 & $59.90 \%$ & 40 & RFS/ OS & Positive $>60 \%$ & $\mathrm{U}$ & Median 39 (24.9 to 49.3$)$ & 2006-2010 \\
\hline Hui LP, 2013 & China & 120 & $47.50 \%$ & 38.3 & OS & $\begin{array}{c}\text { Normal: } 0-1 \text {; } \\
\text { overexpression: } 2-9\end{array}$ & M & Median 30.8 ( 3 to 72 ) & $2001-2010$ \\
\hline Lv TF, 2015 & America & 75 & $96.00 \%$ & 34.7 & OS & $\begin{array}{c}\text { low: } \leq 1 ; \text { overexpression: } \\
\geq 2\end{array}$ & NM & NM & 2004-2009 \\
\hline Zhou Y, 2016 & China & 153 & $17.00 \%$ & 78.4 & OS & low: $<4$; overexpression: $\geq 4$ & $\mathrm{U} / \mathrm{M}$ & Median 57 (4 to 95 ) & 2002-2004 \\
\hline
\end{tabular}

RFS recurrence-free survival; AC adenocarcinoma; U univariate analyses; M multivariate analyses; NM no mention.

not (Follow-up (month) $>36$, pooled HR $=2.476,95 \% \mathrm{CI}$ $=1.728-3.547, P<0.001$; Follow-up (month) $<36 /$ no mention, pooled $\mathrm{HR}=1.731,95 \% \mathrm{CI}=1.045-2.866$, $P=0.033)$.

\section{Association of twist with clinicopathological parameters}

The connections between Twist and clinicopathological parameters are shown in Table 3 and Figure 4. The difference between Twist overexpression and biologically aggressive phenotypes, such as lymph node or other metastasis $(\mathrm{OR}=2.384,95 \% \mathrm{CI}=1.472$ $3.862, P<0.001$, fixed effect) was statistically significant. However, no association was found between Twist and other clinicopathological features, including age $(\mathrm{OR}=$ $1.086,95 \% \mathrm{CI}=0.679-1.736, P=0.731$, fixed effect),
$\operatorname{sex}(\mathrm{OR}=1.104,95 \% \mathrm{CI}=0.726-1.679, P=0.644$, fixed effect), tumor differentiation (OR $=1.981,95 \% \mathrm{CI}=$ $0.996-3.939, P=0.051$, fixed effect), histology type (OR $=0.810,95 \% \mathrm{CI}=0.544-1.206, P=0.299$, fixed effect) and tumor stage $(\mathrm{OR}=1.883,95 \% \mathrm{CI}=0.791-4.485, P$ $=0.153$, random effect).

\section{Sensitivity analysis and publication bias}

The result of the sensitivity analysis is shown in Figure 5. When each independent study was sequentially excluded, the combined $95 \% \mathrm{CI}$ of the remaining four studies did not exceed the $95 \%$ CI of the pooled HR of five studies, indicating that no study dominated the result. Publication bias was tested by HR estimation of the OS. No obvious publication bias was revealed by Begg's test $(P=0.462)$ and Egger's test $(P=0.651)$ (Figure 6).

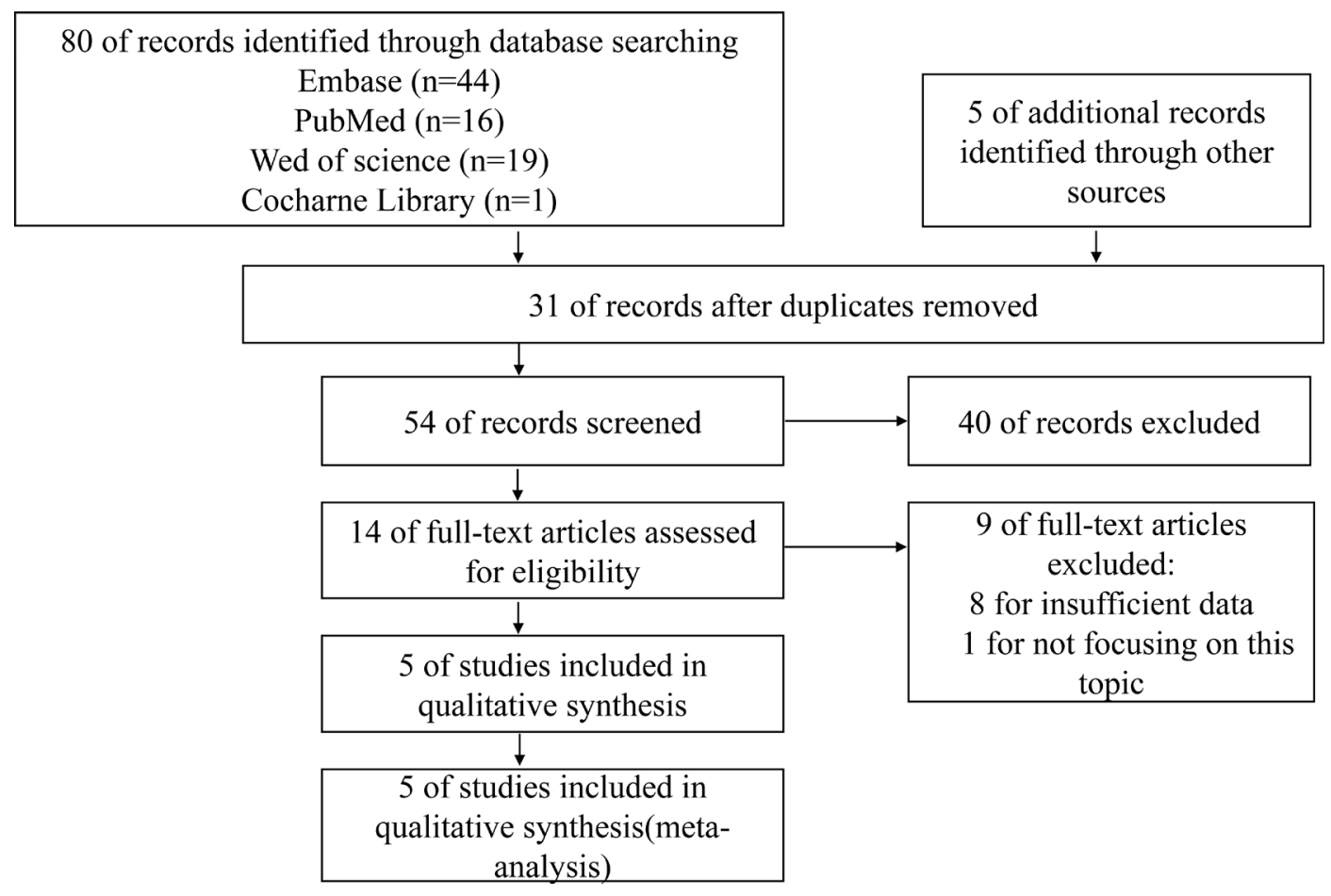

Figure 1: Flow diagram of study selection process. 
Table 2: Meta-analysis of twist overexpression and prognosis in NSCLC

\begin{tabular}{lcccccc}
\hline Categories & Studies (patients) & HR (95\% CI) & $\mathbf{I}^{\mathbf{2}} \mathbf{( \% )}$ & $\mathbf{P}_{\mathrm{H}}$ & $\mathbf{Z}$ & $\mathbf{P}$ \\
\hline RFS & $3(377)$ & $2.476(1.728-3.547)$ & 0.0 & 0.414 & 4.94 & $<0.001$ \\
Univariate analyses & $2(224)$ & $3.219(1.826-5.674)$ & 0 & 0.533 & 4.04 & $<0.001$ \\
Multivariate analyses & $2(273)$ & $1.877(1.268-2.779)$ & 0 & 0.429 & 3.15 & 0.002 \\
Country (China) & $4(497)$ & $2.235(1.619-3.086)$ & 10.8 & 0.339 & 4.89 & $<0.001$ \\
Follow-up (M) $>36$ & $3(410)$ & $2.476(1.728-3.547)$ & 0 & 0.414 & 4.94 & $<0.001$ \\
Follow-up (M) $<36 / \mathrm{NM}$ & $2(162)$ & $1.731(1.045-2.866)$ & 0 & 0.534 & 2.13 & 0.033 \\
\hline
\end{tabular}

All pooled HRs were performed by fixed-effect model.

$\mathrm{P}_{\mathrm{H}} P$-value for heterogeneity based on $Q$ test.

$\mathrm{P} P$-value for statistical significance based on $Z$ test.

\section{DISCUSSION}

NSCLC is the most predominant type of lung cancer and the leading cause of cancer death worldwide. However, there is little by using a combination of metastasis-related markers. EMT is considered to be one of the major molecular mechanisms inducing tumour invasion and metastasis. Twist is a highly conserved bHLH transcription factor that had been found to induce cancers promoting through EMT. There is increasing evidence to support the expression of Twist is associated with worse survival in carcinoma patients, including lung cancer [35]. In vivo and in vitro studies were implemented to gain a comprehensive analysis of Twist in NSCLC patients, animal models, and human NSCLC cell lines. Twist and related signal transduction pathways play important roles in carcinoma progression and may serve as potential target for treating carcinoma [37]. Twist is a significant prognostic marker to predict overall survival in patients with lung adenocarcinoma [25]. The findings supported that Twist expression was linked to EGFR mutations by collaborating with the EGF pathway in promoting EMT in EGFR mutated lung adenocarcinoma [36]. Twist is associated with hypoxic metastasis and EMT of NSCLC and serves as a potential therapeutic target in hypoxic lung cancer $[35,38]$. Moreover, Twist is correlated
Study

ID

Hung, J. J, 2009

Jiang W,2012

Hui LP,2013

Lv TF, 2015

Zhou Y,2016

Overall (I-squared $=0.0 \%, p=0.488$ )
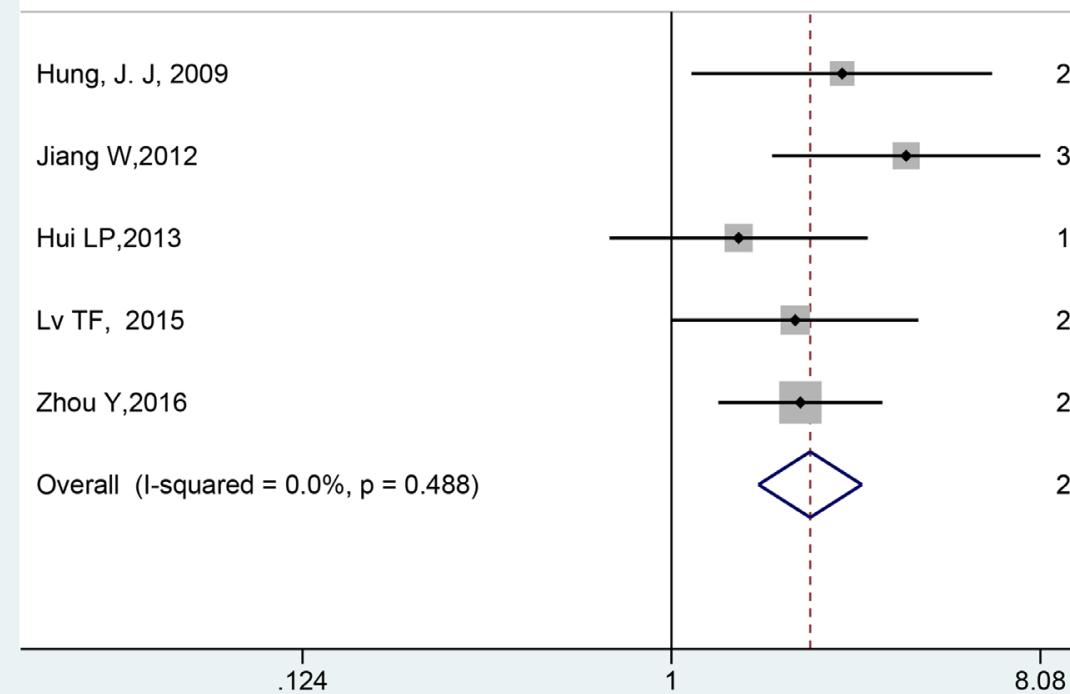

$\mathrm{HR}(95 \% \mathrm{Cl})$

$\%$

Weight

$2.63(1.12,6.15) \quad 11.82$

$3.78(1.77,8.08) \quad 14.84$

$1.46(0.70,3.04) \quad 16.03$

$2.02(1.00,4.05) \quad 17.63$

$2.08(1.30,3.30) \quad 39.69$

$2.19(1.64,2.94) \quad 100.00$

Figure 2: Forest plot of the correlation between twist and OS in NSCLC patients. 
Table 3: Meta-analysis of Twist overexpression and clinicopathological features in NSCLC

\begin{tabular}{lcccccc}
\hline Categories & Studies (patients) & OR (95\% CI) & $\mathbf{I}^{\mathbf{2}} \mathbf{( \% )}$ & $\mathbf{P}_{\mathrm{H}}$ & $\mathbf{Z}$ & $\mathbf{P}$ \\
\hline Age $(>60 / \leq 60)$ & $3(348)$ & $1.086(0.679-1.736)$ & 0 & 0.857 & 0.34 & 0.731 \\
Sex (Male / Female) & $5(572)$ & $1.104(0.726-1.679)$ & 47.0 & 0.109 & 0.46 & 0.644 \\
Tumor differentiation & $3(348)$ & $1.981(0.996-3.939)$ & 0 & 0.435 & 1.95 & 0.051 \\
(Moderate, Poor / Well) & & & & & & \\
Lymph node or other metastasis & $3(348)$ & $2.384(1.472-3.862)$ & 0 & 0.411 & 3.53 & $<0.001$ \\
Histology (adenocarcinoma/non- & $5(572)$ & $0.810(0.544-1.206)$ & 22.0 & 0.274 & 1.04 & 0.299 \\
adenocarcinoma) & & & & & & \\
Tumor stage (II, III, IV/I) & $4(435)$ & $1.883(0.791-4.485)$ & 70.6 & 0.017 & 1.43 & 0.153 \\
\hline
\end{tabular}

All pooled ORs were performed by fixed-effect model except for tumor stage with random-effect model.

OR odds rate

$\mathrm{P}_{\mathrm{H}} P$-value for heterogeneity based on $Q$ test.

P $P$-value for statistical significance based on $Z$ test.

with other co-expression markers (p-4E-BP1, HIF-1 $\alpha$, Snail, N-cadherin, Foxc2, Slug) in predicting the worse prognostic outcome of NSCLC [27, 29-31]. Sufficient data have shown a significant correlation between Twist expression and poor prognosis of NSCLC patients. However, controversy exists concerning the correlation between Twist and prognostic value with respect to NSCLC.

In this study, we meta-analyzed the literature on Twist expression in NSCLC and its association with OS and clinicopathological features. Results showed that Twist overexpression was correlated with poor OS and RFS. All subgroup analysis demonstrated the adverse role of Twist overexpression in NSCLC. Moreover, the sensitivity analysis indicated that our results were relatively conclusive. Thus, Twist expression may be an independent prognostic factor of OS. We also found a significant association between Twist overexpression and poor clinicopathological features, such as metastasis including lymph node metastasis. This study is a literature-based analysis, Begg's funnel plot and Egger's test
Study

ID
$\%$

$\operatorname{HR}(95 \% \mathrm{Cl})$

Zhou Y,2016

Overall (I-squared $=0.0 \%, p=0.414)$

NOTE: Weights are from random effects analysis

124
$2.63(1.12,6.15)$

17.81

22.37

59.82

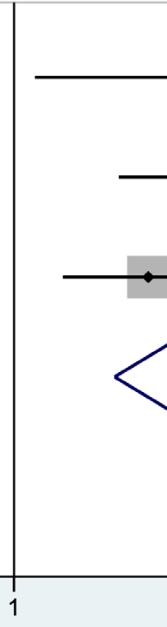

$2.08(1.30,3.30)$

$2.48(1.73,3.55)$

100.00
$3.78(1.77,8.08)$

Figure 3: Forest plot of the correlation between twist and RFS in NSCLC patients. 
were used to assess publication bias. All five eligible studies yielded a Begg's test score of $P=0.462$ and Egger's test score of $P=0.651$. These results suggested that there was no publication bias.

Although this meta-analysis aimed to provide the best possible estimate of the clinical significance of Twist expression, it still had limitations. First of all, all the included studies were retrospective, which might increase

\section{A}

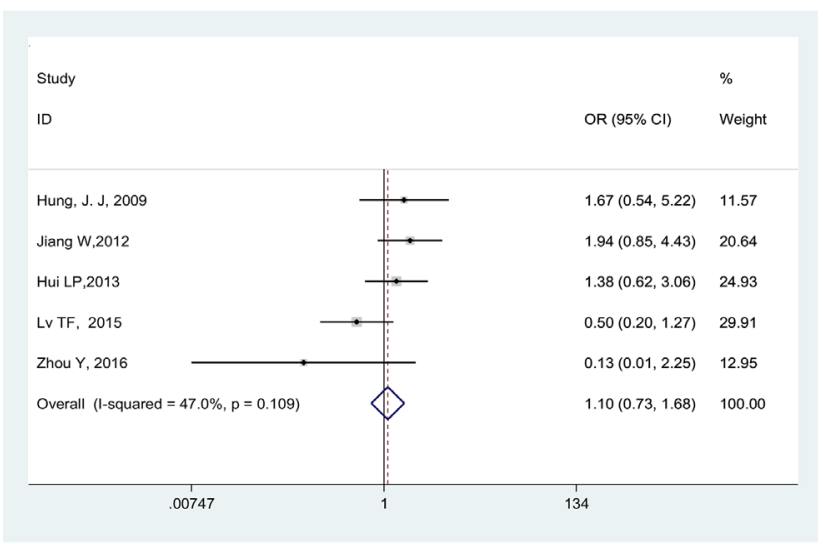

C

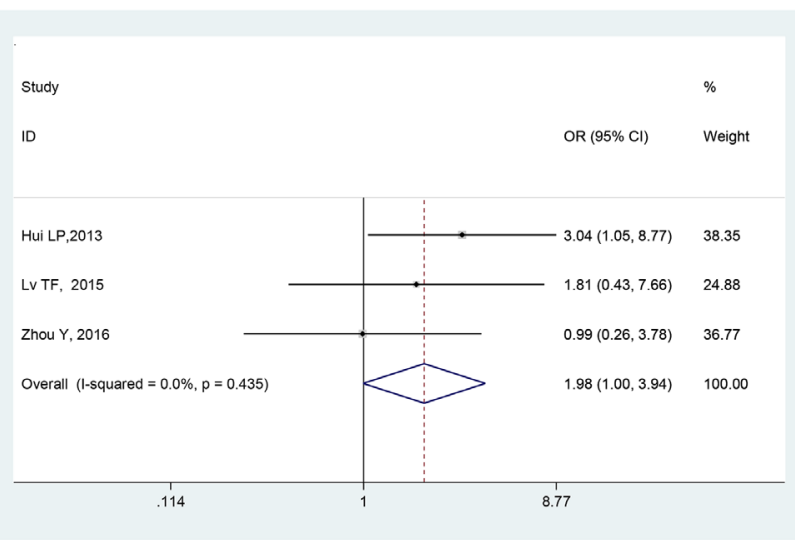

$\mathrm{E}$

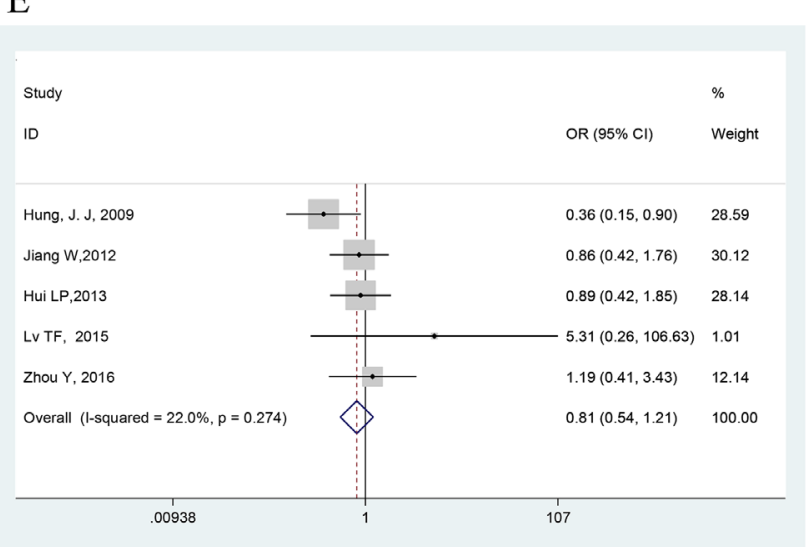

the bias of our study. Secondly, the sample size of included articles was relatively small with only 572 cases. Thirdly, no methods and cutoff definitions had been accepted and validated for evaluating Twist expression. Finally, although publication bias was not occurred in this study, since most of including studies were from China, the researchers tended to publish unusually high proportion of positive results [39].

B

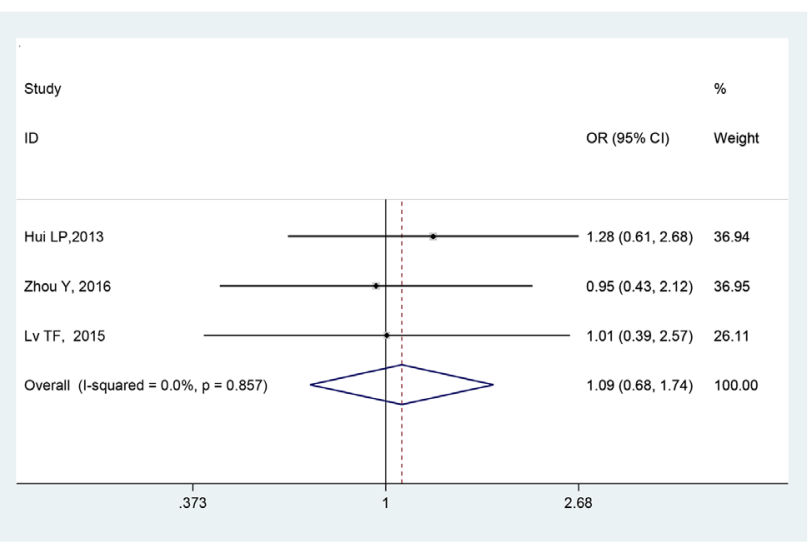

D

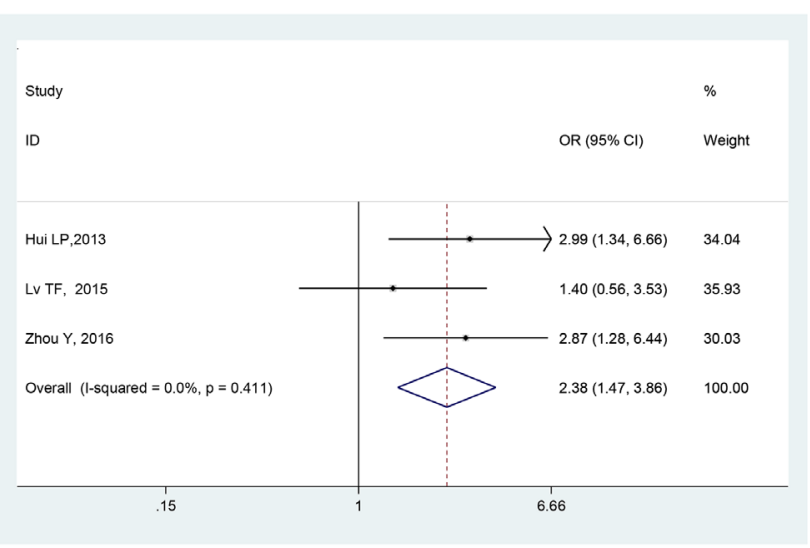

$\mathrm{F}$

Study

ID
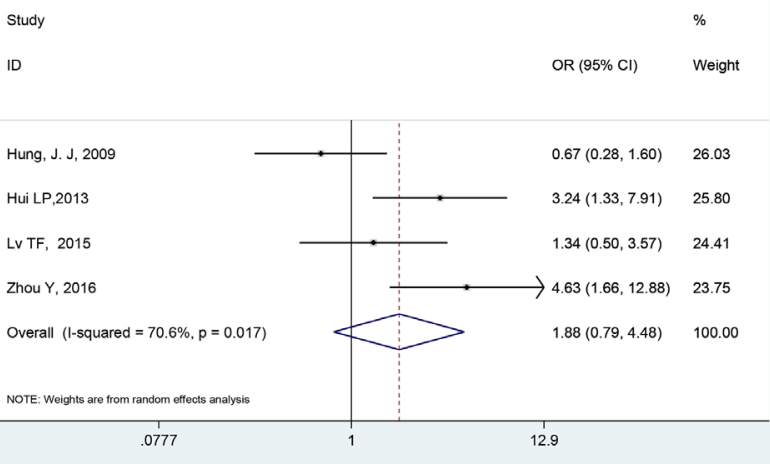

Figure 4: Forest plots showing the OR of Twist overexpression vs. normal Twist expression for clinicopathological features. (A) Age; (B) Sex; (C) Tumor differentiation; (D) Lymph node or other metastasis; (E) Histology; (F) Tumor stage. All pooled ORs were performed by fixed-effect model except for tumor stage with (NOTE: Weights are from random effects analysis). The difference between Twist overexpression and lymph node or other metastasis was statistically significant $(\mathrm{OR}=2.384, P<0.001)$. 
In conclusion, despite the limitations listed above, this meta-analysis showed that Twist overexpression is correlated with poor OS and clinicopathological features in NSCLC, suggesting that the Twist may be a poor prognostic factor and therapeutic target in NSCLC. Subgroup analysis revealed that the expression of Twist

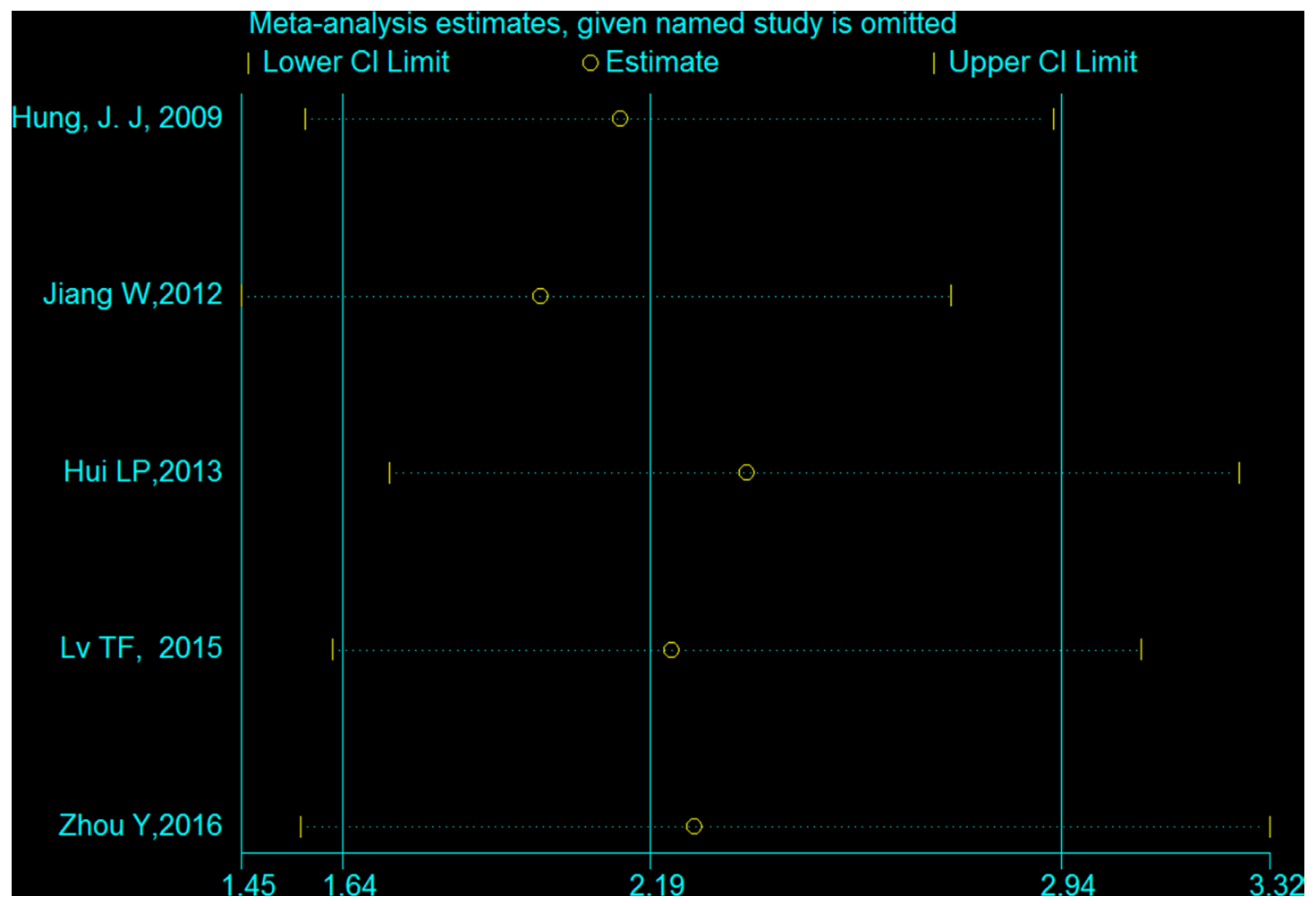

Figure 5: Effect of individual studies on the pooled HR for Twist overexpression and OS of NSCLC. The horizontal axis number 2.19 represents the overall HR, and the 1.64 and 2.94 represent the $95 \% \mathrm{CI}$.

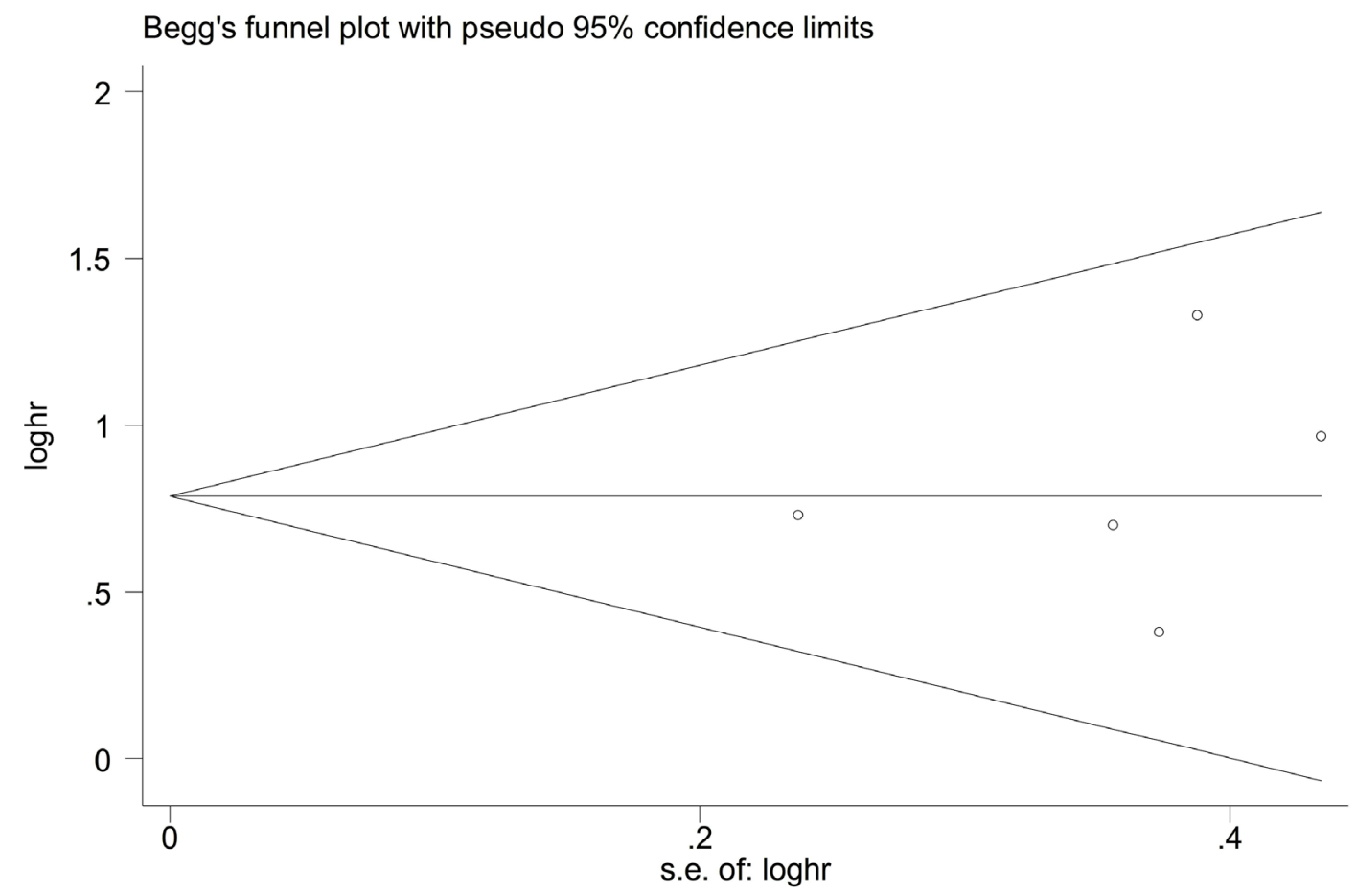

Figure 6: Funnel plot analysis to detect publication bias. There was no publication bias $(P=0.462)$. 
in Chinese patients may be more closely associated with the prognosis of NSCLC than in other countries. Moreover, further larger well-designed prospective studies employing a standard evaluated method are needed to confirm these findings from our study.

\section{MATERIALS AND METHODS}

Meta-analysis was conducted in accordance with the preferred reporting items for meta-analysis criteria [40].

\section{Literature search strategy}

We performed a complete computer-based search of the PubMed, Embase, web of science and the Cochrane Library databases for clinical trials in original articles up to the date of July 10, 2017. The search strategy was conducted according to a combination of the following terms: "((nonsmall cell lung cancer) OR NSCLC) AND ((Twist) OR TWIST) AND ((()(prognosis) OR outcome) OR survival) OR prognostic value) OR prognostic biomarker)". The irrelevant articles were directly excluded by scanning the titles or abstracts. We also examined reference lists from the adopted studies in order to avoid missing studies which may fulfill our requirements. The remaining articles were then reviewed comprehensively by reading the full text.

\section{Inclusion criteria}

Studies meeting all the following criteria were included: 1) retrospective or prospective studies; 2) paying attention to the relationship between Twist expression and survival in NSCLC; 3) providing enough data to get the HR for prognosis outcomes, along with their $95 \%$ CIs or $P$ values; 4) published in English.

\section{Exclusion criteria}

The exclusion criteria were as followed: 1) neither the retrospective nor prospective studies; 2) studies without sufficient data to pool the HR; 3) studies not focusing on the role of the Twist expression on the prognosis in NSCLC; 4) not published in English; 5) were reviews, case reports, conference abstracts or comments, or studies in vitro or in animal models.

\section{Data abstraction}

Two investigators (Meng $\mathrm{Li}$ and Zhang $\mathrm{X}$ ) independently reviewed all the manuscripts. The following data were abstracted: first name of the author, publication year, country of the study, sample size, histology type, positive rate of Twist, outcome, cut-off value of Twist expression, survival analysis, follow-up time and recruitment time. The HRs of RFS or OS obtained directly from published articles. The HR assessed by multivariate analysis was abstracted when the multivariate analysis and univariate analysis were both provided. All HR and 95\% CI were reported in these studies.

\section{Quality assessment}

The Newcastle-Ottawa Quality Assessment Scale (NOS) was applied to assess the quality of the selected studies. The NOS included three main aspects: selection, comparability, and outcome [41, 42]. And NOS score $\geq 6$ is considered high-quality study. Discrepancy was resolved by consulting.

\section{Statistical analysis}

Pooled analyses were carried out by STATA software (version 14.0; StataCorp, College Station, Texas, USA). The prognosis outcomes were explored using the HR and the corresponding 95\% CI. The prognosis outcomes mainly contained the OS or RFS. The heterogeneity was assessed by Cochran's $Q$ test and Higgins $\mathrm{I}^{2}$ significant when $P<0.05$ and/or $\mathrm{I}^{2}$ [43]. When the heterogeneity was $>50 \%$, the random effect model was used; if not, the fixed-effects model was applied. Stata 14.0 was used to assess the robustness of the results. All the P was twoside and $P<0.05$ was considered statistically significant. Visual inspection of Begg's test and Egger's test [44] were used to evaluate publication bias $(P<0.01$ was considered statistically significant). The sensitivity analysis was conducted by using the "metaninf" STATA command (sequential exclusion of each individual study then pooled HR).

\section{Abbreviations}

NSCLC $=$ non-small cell lung cancer, OS $=$ overall survival, RFS $=$ recurrence-free survival, 95\% confidence interval $=95 \% \mathrm{CI}$, EMT $=$ Epithelial to mesenchymal transition, $\mathrm{HR}=$ hazard ratio, $\mathrm{OR}=$ odds rate, $\mathrm{bHLH}$ = basic helix-loop-helix, Newcastle-Ottawa Quality Assessment Scale $=$ NOS.

\section{Author contributions}

Study concepts and design: Meng Li, Xing Zhang; Literature search: Meng Li, Xing Zhang; Data extraction: Jiubin $\mathrm{Wu}$, Kaiwen Hu; Statistical analysis and quality assessment: Xiaoqing $\mathrm{Xu}$, Xiuwei Guo; Tables and figures: Meng Li, Xing Zhang and Xiaoqing $\mathrm{Xu}$; Manuscript preparation and revision: Meng Li, Xing Zhang and Peitong Zhang. All authors have participated sufficiently in the study and approved the final version.

\section{CONFLICTS OF INTEREST}

The authors have declared no conflicts of interest. 


\section{FUNDING}

This work was supported by the National Nature Science Foundation of China (No.81673797).

\section{REFERENCES}

1. Siegel RL, Miller KD, Jemal A. Cancer statistics, 2016. CA Cancer J Clin. 2016; 66:7-30.

2. Chen W, Zheng R, Baade PD, Zhang S, Zeng H, Bray F, Jemal A, Yu XQ, He J. Cancer statistics in China, 2015. CA Cancer J Clin. 2016; 66:115-132.

3. Esposito L, Conti D, Ailavajhala R, Khalil N, Giordano A. Lung Cancer: Are we up to the Challenge? Current Genomics. 2010; 11:513-518.

4. Park E, Park SY, Sun PL, Jin Y, Kim JE, Jheon S, Kim K, Lee CT, Kim H, Chung JH. Prognostic significance of stem cellrelated marker expression and its correlation with histologic subtypes in lung adenocarcinoma. Oncotarget. 2016; 7:42502-42512. https://doi.org/10.18632/oncotarget.9894.

5. Nieto MA. Epithelial plasticity: a common theme in embryonic and cancer cells. Science. 2013; 342:1234850.

6. Pradella D, Naro C, Sette C, Ghigna C. EMT and stemness: flexible processes tuned by alternative splicing in development and cancer progression. Molecular cancer. 2017; 16:8.

7. Birchmeier $\mathrm{W}$, Behrens J. Cadherin expression in carcinomas: role in the formation of cell junctions and the prevention of invasiveness. Biochimica et Biophysica Acta 1994; 1198:11-26.

8. Peinado H, Cano A. New potential therapeutic targets to combat epithelial tumor invasion. Clin Transl Oncol. 2006; 8:851-857.

9. Yang $\mathrm{MH}, \mathrm{Wu} \mathrm{KJ}$. TWIST activation by hypoxia inducible factor-1 (HIF-1): implications in metastasis and development. Cell Cycle. 2008; 7:2090-2096.

10. Morizane R, Fujii S, Monkawa T, Hiratsuka K, Yamaguchi $\mathrm{S}$, Homma K, Itoh H. miR-363 induces transdifferentiation of human kidney tubular cells to mesenchymal phenotype. Clin Exp Nephrol. 2016; 20:394-401.

11. Rodriguez Y, Gonzalez-Mendez RR, Cadilla CL. Evolution of the Twist Subfamily Vertebrate Proteins: Discovery of a Signature Motif and Origin of the Twist1 Glycine-Rich Motifs in the Amino-Terminus Disordered Domain. PLoS One. 2016; 11:e0161029.

12. Qin Q, Xu Y, He T, Qin C, Xu J. Normal and diseaserelated biological functions of Twist1 and underlying molecular mechanisms. Cell Res. 2012; 22:90-106.

13. Franco HL, Casasnovas J, Rodriguez-Medina JR, Cadilla CL. Redundant or separate entities?--roles of Twist1 and Twist2 as molecular switches during gene transcription. Nucleic Acids Res. 2011; 39:1177-1186.

14. Ansieau S, Bastid J, Doreau A, Morel AP, Bouchet BP, Thomas C, Fauvet F, Puisieux I, Doglioni C, Piccinin S, Maestro R, Voeltzel T, Selmi A, et al. Induction of EMT by twist proteins as a collateral effect of tumor-promoting inactivation of premature senescence. Cancer cell. 2008; 14:79-89.

15. Li L, Cserjesi P, Olson EN. Dermo-1: A Novel TwistRelated bHLH Protein Expressed in the Developing Dermis. Dev Biol. 1995; 172:280-292.

16. Ansieau S, Morel AP, Hinkal G, Bastid J, Puisieux A. TWISTing an embryonic transcription factor into an oncoprotein. Oncogene. 2010; 29:3173-3184.

17. Peinado H, Olmeda D, Cano A. Snail, Zeb and bHLH factors in tumour progression: an alliance against the epithelial phenotype? Nat Rev Cancer. 2007; 7:415-428.

18. Tan EJ, Thuault S, Caja L, Carletti T, Heldin CH, Moustakas A. Regulation of transcription factor Twist expression by the DNA architectural protein high mobility group A2 during epithelial-to-mesenchymal transition. J Biol Chem. 2012; 287:7134-7145.

19. Eckert MA, Lwin TM, Chang AT, Kim J, Danis E, OhnoMachado L, Yang J. Twist1-induced invadopodia formation promotes tumor metastasis. Cancer cell. 2011; 19:372-386.

20. Zhang YQ, Wei XL, Liang YK, Chen WL, Zhang F, Bai JW, Qiu SQ, Du CW, Huang WH, Zhang GJ. Over-Expressed Twist Associates with Markers of Epithelial Mesenchymal Transition and Predicts Poor Prognosis in Breast Cancers via ERK, Akt Activation. PLoS One. 2015; 10:e0135851.

21. Chuang HY, Jiang JK, Yang MH, Wang HW, Li MC, Tsai CY, Jhang YY, Huang JC. Aminopeptidase A initiates tumorigenesis and enhances tumor cell stemness via TWIST1 upregulation in colorectal cancer. Oncotarget. 2017; 8:21266-21280. https://doi.org/10.18632/oncotarget.15072.

22. Chen S, Chen JZ, Zhang JQ, Chen HX, Yan ML, Huang L, Tian YF, Chen YL, Wang YD. Hypoxia induces TWIST-activated epithelial-mesenchymal transition and proliferation of pancreatic cancer cells in vitro and in nude mice. Cancer letters. 2016; 383:73-84.

23. da Silva SD, Alaoui-Jamali MA, Soares FA, Carraro DM, Brentani HP, Hier M, Rogatto SR, Kowalski LP. TWIST1 is a molecular marker for a poor prognosis in oral cancer and represents a potential therapeutic target. Cancer. 2014; 120:352-362.

24. Miura N, Yano T, Shoji F, Kawano D, Takenaka T, Ito K, Morodomi Y, Yoshino I, Maehara Y. Clinicopathological significance of Sip1-associated epithelial mesenchymal transition in non-small cell lung cancer progression. Anticancer Research. 2009; 29:4099-4106.

25. Shi YL, Wu HY, Zhang MY, Ding L, Meng FQ, Fan XS. Expression of the epithelial-mesenchymal transitionrelated proteins and their clinical significance in lung adenocarcinoma. Diagn Pathol. 2013; 8.

26. Lee JH, Park WY, Jeong SM, Lee MK, Kim YD, Shin DH, Lee CH. Relationship between the Endogenous Hypoxic Markers Hypoxia Inducible Factor-1 alpha, Carbonic Anhydrase IX, and Epithelial Mesenchymal Transition Regulator TWIST Expression in Non-small Cell Lung Cancer. Korean Journal of Pathology. 2010; 44:469-476. 
27. Lv TF, Wang Q, Cromie M, Liu HB, Tang S, Song Y, Gao WM. Twist1-mediated 4E-BP1 regulation through mTOR in non-small cell lung cancer. Oncotarget. 2015; 6:3300633018. https://doi.org/10.18632/oncotarget.5026.

28. Zhou Y, Liao Q, Han Y, Chen J, Liu Z, Ling H, Zhang J, Yang W, Oyang L, Xia L, Wang L, Wang H, Xue L, et al. Rac1 overexpression is correlated with epithelial mesenchymal transition and predicts poor prognosis in nonsmall cell lung cancer. J Cancer. 2016; 7:2100-2109.

29. Hui L, Zhang S, Dong X, Tian D, Cui Z, Qiu X. Prognostic significance of twist and N-cadherin expression in NSCLC. PLoS One. 2013; 8:e62171.

30. Jiang W, Pang XG, Wang Q, Shen YX, Chen XK, Xi JJ. Prognostic role of Twist, Slug, and Foxc2 expression in stage i non-small-cell lung cancer after curative resection. Clinical Lung Cancer. 2012; 13:280-287.

31. Hung JJ, Yang MH, Hsu HS, Hsu WH, Liu JS, Wu KJ. Prognostic significance of hypoxia-inducible factor-1alpha, TWIST1 and Snail expression in resectable non-small cell lung cancer. Thorax. 2009; 64:1082-1089.

32. Parmar MK, Torri V, Stewart L. Extracting summary statistics to perform meta-analyses of the published literature for survival endpoints. Statist Med. 1998; 17:2815-2834.

33. Tierney JF, Stewart LA, Ghersi D, Burdett S, Sydes MR. Practical methods for incorporating summary time-to-event data into meta-analysis. Trials. 2007; 8:16.

34. Zeng J, Zhan P, Wu G, Yang W, Liang W, Lv T, Song Y. Prognostic value of Twist in lung cancer: systematic review and meta-analysis. Translational lung cancer research. 2015; 4:236-241.

35. Wushou A, Hou J, Zhao YJ, Shao ZM. Twist-1 up-regulation in carcinoma correlates to poor survival. Int J Mol Sci. 2014; 15:21621-21630.

36. Pallier K, Cessot A, Cote JF, Just PA, Cazes A, Fabre E, Danel C, Riquet M, Devouassoux-Shisheboran M, Ansieau S, Puisieux A, Laurent-Puig P, Blons H. TWIST1 a new determinant of epithelial to mesenchymal transition in
EGFR mutated lung adenocarcinoma. PLoS One. 2012; 7:e29954.

37. Zhang D, Sun B, Zhao X, Ma Y, Ji R, Gu Q, Dong X, Li J, Liu F, Jia X, Leng X, Zhang C, Sun R, Chi J. Twist1 expression induced by sunitinib accelerates tumor cell vasculogenic mimicry by increasing the population of CD133+ cells in triple-negative breast cancer. Molecular cancer. 2014; 13:207.

38. Wei L, Sun JJ, Cui YC, Jiang SL, Wang XW, Lv LY, Xie L, Song XR. Twist may be associated with invasion and metastasis of hypoxic NSCLC cells. Tumour biology. 2016; 37:9979-9987.

39. Vickers A, Goyal N, Harland R, Rees R. Do Certain Countries Produce Only Positive Results? A Systematic Review of Controlled Trials. Controlled Clin Trials. 1998; 19:159-166.

40. Liberati A, Altman DG, Tetzlaff J, Mulrow C, Gotzsche PC, Ioannidis JP, Clarke M, Devereaux PJ, Kleijnen J, Moher D. The PRISMA statement for reporting systematic reviews and meta-analyses of studies that evaluate health care interventions: explanation and elaboration. Annals of internal medicine. 2009; 151:W65-94.

41. Maxwell L, Santesso N, Tugwell PS, Wells GA, Judd M, Buchbinder R. Method guidelines for Cochrane Musculoskeletal Group systematic reviews. The Journal of rheumatology. 2006; 33:2304-2311.

42. Wells GA, Shea B, O'Connell D, Peterson J, Welch V, Losos M, Tugwell P. The Newcastle-Ottawa Quality Assessment Scale(NOS) for assessing the quality of nonrandomized studies in meta-analyses. Ottawa Health Research Institute Web site.

43. Higgins JP, Thompson SG, Deeks JJ, Altman DG. Measuring inconsistency in meta-analyses. BMJ. 2003; 327:557-560.

44. Egger M, Davey Smith G, Schneider M, Minder C. Bias in meta-analysis detected by a simple, graphical test. BMJ. 1997; 315:629-634. 